\title{
Aptitude à la multiplication de Pseudocedrela kotschyi (Schweinf.) Harms par graines et par boutures de tige et de racine au Bénin
}

\author{
Aymar Guy DEGUENONVO ${ }^{1 *}$, Justin DOSSOU ${ }^{2}$ et Rodrigue IDOHOU ${ }^{1}$ \\ ${ }^{1}$ Laboratoire de Biomathématiques et d'Estimations Forestières (LABEF) www.labef-uac.org , Faculté des \\ Sciences Agronomiques de l'Université d'Abomey-Calavi, Po Box 1525 Cotonou, Bénin. \\ ${ }^{2}$ Laboratoire d'Études et de Recherches Forestières (LERF) http://wwwlerf-up.com, Faculté d'Agronomie, \\ Université de Parakou, BP 123 Parakou, Bénin. \\ *Auteur correspondant ; E-mail : aymardeg@yahoo.fr ; Tél : (+229) 97683153
}

\section{RESUME}

Pseudocedrela kotschyi, fait partie des espèces les plus exploitées en bois d'œuvre, en charbon de bois et d'autres utilisations au Bénin. Face à cette exploitation, elle est confrontée au problème de régénération naturelle. Pour une gestion durable de cette espèce, il est impérieux de procéder à la régénération assistée. L'objectif de cette étude était de ressortir les voies de multiplication de l'espèce. Pour cela, un test de germination a été fait sur 200 graines sans traitement prégerminatif. Une reproduction végétative par tige et par racine a été faite sur 92 boutures selon deux classes de diamètre ([0 cm à $1,5 \mathrm{~cm}]$ et $[1,5 \mathrm{~cm}$ à $3 \mathrm{~cm}])$. Les résultats obtenus montrent que le taux de germination des graines de $P$. kotschyi est de $84 \%$ sur une durée de 12 jours. La grosseur et le type de bouture ont un effet significatif $(\mathrm{P}<0,05)$ sur le bourgeonnement, débourrement et le suivi des bourgeons. Les boutures de racine de grand diamètre ont donné les moyennes les plus élevées soit 10,62 bougeons avec 6,12 bourgeon débourré par bouture avec un taux de suivi de 47,82\%. Il ressort que $P$. kotschyi peut se multiplier par graines et par boutures de racine.

(C) 2020 International Formulae Group. All rights reserved.

Mot clés : Multiplication, reboisement, bouturage, germination, Pseudocedrela kotschyi.

\section{Ability to propagate Pseudocedrela kotschyi (Schweinf.) Harms by seeds and by stem and root cuttings in Benin}

\begin{abstract}
Pseudocedrela kotschyi, is one of the most exploited species in timber, charcoal and other uses in Benin. Faced with this exploitation, it is faced with the problem of natural regeneration. For sustainable management of this species, it is imperative to carry out assisted regeneration. The objective of this study was to highlight the ways in which the species multiplies. For this porpose, a germination test was done on 200 seeds without pregerminative treatment. A vegetative reproduction by stem and by root was made on 92 cuttings according to two classes of diameter ([0 $\mathrm{cm}$ to $1.5 \mathrm{~cm}]$ and $[1.5 \mathrm{~cm}$ to $3 \mathrm{~cm}])$. The results obtained show that the germination rate of $P$. kotschyi seeds is $84 \%$ over a period of 12 days. The size and type of cuttings have a significant effect
\end{abstract}


( $\mathrm{P}<0.05$ ) on budding, budding and monitoring of buds. Large diameter root cuttings gave the highest averages, ie 10.62 buds with 6.12 buds budded by cuttings with a follow-up rate of $47.82 \%$. It appears that $P$. kotschyi can be propagated by seeds and by root cuttings.

(C) 2020 International Formulae Group. All rights reserved.

Keywords: Multiplication, reforestation, cuttings, germination, Pseudocedrela kotschyi.

\section{INTRODUCTION}

En Afrique, les forêts claires sont les résultats d'une dégradation des forêts denses sèches et elles se maintiennent dans cet état du fait des feux de brousse et de l'existence d'une saison sèche suffisamment longue (Bellefontaine et al., 2000). Ces forêts claires fournissent de très nombreuses ressources animales et végétales qui sont des sources d'alimentation, de médicaments, de fourniture en bois d'énergie et de bois d'œuvre pour les populations locales (Goussanou et al., 2011). Ces forêts tropicales et subtropical ont été détruits à 7 millions d'hectare par an entre les années 2000 et 2010, avec pour conséquence la disparition des espèces végétales de leurs écosystèmes naturels (Adomou et al., 2017). Malgré cette disparition des forêts chaque année, l'Afrique dispose toujours d'une diversité biologique très élevée, à tel point que l'avenir de notre planète dépend de sa survie (Avikpo et al., 2017).

Au Bénin, l'agriculture, la fabrication de charbon, la recherche du bois énergie et du bois d'œuvre justifient le fort taux de déforestation (MEPN, 2009). En effet, la consommation du charbon de bois des ménages a été estimée à 2,6\% en milieu rural et 36,5\% en milieu urbain (PBF II/IFN, 2007). La population urbaine constitue donc le grand consommateur de charbon de bois à cause de la qualité de son combustible, sa manipulation facile, et son acquisition à petite quantité au fur et à mesure de ses besoins (Yaoitcha et al., 2007). Les essences forestières exploitées à cet effet sont : Pseudocedrela kotschyi (Schweinf.) Harms Anogeissus leiocarpa (DC.) Guill. et Perr.; Prosopis africana (Guill et Perr) Taub.; Burkea africana Hook. et Pterocarpus erinaceus Poir. Celles-ci sont devenues de plus en plus rares (Agbani et al., 2004). À part Diospyros mespiliformis qui présente un taux de régénération relativement élevé $(8,9 \%)$, les autres espèces de valeur telles qu'Afzelia africana, Pterocarpus erinaceus, Pseudocedrela kotschyi et Khaya senegalensis montrent une régénération faible et ne représentent qu'environ $5 \%$ du total de la régénération (Agbani et al., 2004).

Pseudocedrela kotschyi est un arbre de la famille des Méliacées répandu dans les savanes soudaniennes et guinéennes du Sénégal au Cameroun, jusqu'au Soudan sur des sols lourds et mal drainés. Une répartition irrégulière, localement commune et grégaire d'après les travaux de Abonnier (2009). C'est un arbre au voisinage de 20 mètres haut avec un large couronné à branches fissurées et fleurs blanches parfumées. $P$. kotschyi est fortement exploité à cause de son importance sur le plan médicinal. Selon Ojewole en 2003, les feuilles sont utilisées pour lutter contre le diabète les écorces du tronc de $P$. kotschyi sont utilisées comme Anti-inflammatoires, antidysentériques. La racine est utilisée comme un fébrifuge dans le traitement des maladies gastro-intestinales et rhumatisme. La racine de $P$. kotschyi est aussi utilisée dans le traitement d'helminthiase intestinale (Koné et al., 2004). Il a été établi que la racine et les branches de $P$. kotschyi sont largement utilisés comme brosse végétale des dents (Okunade et al., 2007; Kassim et al., 2009).

Malgré ces utilisations, Pseudocedrela kotschyi est menacé dans son habitat naturel par plusieurs actions anthropiques. Au Bénin, Pseudocedrela kotschyi est fréquent dans la zone soudanienne et surtout exploité pour son rendement élevé en charbon (Yaoitcha et al., 2016). La densité de régénération est de 2 individus à l'hectare pour les diamètres à hauteur d'homme compris entre $1 \mathrm{~cm}$ et $5 \mathrm{~cm}$ et 0 individus à 1 'hectare pour les diamètres à hauteur d'homme compris entre $5 \mathrm{~cm}$ et $10 \mathrm{~cm}$ au Nord du Bénin (Sokpon et al., 2006). Parmi les cortèges des espèces exploitées pour la 
production du charbon, les principales souches d'espèces rencontrées sont: Burkea africana, Prosopis africana, Anogeissus leiocarpa, Pterocarpus erinaceus, Vitellaria paradoxa et Pseudocedrela kotschyi (Yaoitcha et al., 2016). Ces espèces autrefois très communes, ont été de nos jours décimées et sont devenues très rares dans les formations naturelles. $\mathrm{La}$ restriction de l'exploitation de Pseudocedrela kotschyi est très capitale à cause de sa faible probabilité de reconstitution (Sokpon et al., 2006).

L'aménagement durable des forêts
claires du Bénin l'enrichissement des peuplements ainsi que la mise en cuvre de mesures restrictives concernant l'exploitation de certaines espèces telles que Pterocarpus erinaceus, Pseudocedrela kotschyi, Daniellia oliveri et Diospyros mespiliformis. Pour la pérennisation de l'espèce dans les fonctions socioéconomiques et le maintien de la diversité, il est nécessaire de penser à la régénération pour parfaire l'aménagement de nos forêts. Cette étude se focalise donc sur l'essai de multiplication par graines et par boutures de tiges et de racines de $P$. kotschyi afin de doter aux communautés une base de sa multiplication pour les reboisements forestiers.

\section{MATERIEL ET METHODES}

\section{Description du site d'expérimentation}

Ces travaux de multiplication ont été conduits à la ferme d'application de la Faculté d'Agronomie de l'Université de Parakou au Bénin situé à $9^{\circ} 21^{\prime}$ de latitude Nord, à $2^{\circ} 36^{\prime}$ de longitude Est à une altitude moyenne de 350 $m$ et présente un relief assez modeste. La ville de Parakou est la Capitale régionale du Nord Bénin située au centre de la République du Bénin à $407 \mathrm{~km}$ de Cotonou. Le climat de ce milieu d'étude est de type tropical humide (climat Sud soudanien) qui est caractérisé par l'alternance d'une saison de pluies (mai à octobre) et d'une saison sèche (novembre à avril). C'est en décembre-janvier que l'on enregistre les températures les plus basses à Parakou. La précipitation moyenne annuelle est de $1200 \mathrm{~mm}$ dont le maximum survient entre juillet, août et septembre. Elle se singularise sur le plan pédologique par la prédominance des sols à texture légère, d'épaisseur importante due à la faiblesse de l'érosion. La faiblesse de l'érosion entraîne un lessivage en profondeur important.

\section{Matériels}

Le matériel utilisé est composé principalement des graines, boutures de tiges et de racines de $P$. kotschyi. Les pots en polyéthylène biodégradable ont été utilisés pour contenir le sol forestier. D'autres matériels ont été utilisés à savoir, arrosoir, un appareil photo numérique pour la prise des photos et un ordinateur portatif.

\section{Test de germination des graines de $P$. kotschyi}

Les graines utilisées pour ces essais proviennent de la population naturelle de $P$. kotschyi dans la forêt classée de Dogo dans la commune de Kétou. Elles ont été conservées pendant deux mois après séchage et dans un bocal perforé de trous fin pour favoriser la respiration des graines jusqu'au mois du démarrage des travaux. Au total, 200 graines ont été utilisées. Elles ont été semées dans les pots en polyéthylène biodégradable. Le temps de latence ( $\mathrm{Tl})$ et le taux de germination $(\mathrm{Tg})$ des graines de $P$. kotschyi ont été les paramètres étudiés.

$>$ Le taux de germination $(\mathrm{Tg})$ a été déterminé par le nombre de graines germées (Ngg) par rapport au nombre de graines semées (Ngs). $T g=\frac{N g g}{N g s} X 100$

$>$ Durée moyenne de germination: le nombre de jours entre le jour de semis et le jour de la dernière germination des graines.

$>$ Temps de latence (dormance) des graines : le nombre de jours avant la première germination.

\section{Bouture de tige et de racine}

Les boutures de tiges et de racines ont été prélevées sur des arbres de $P$. kotschyi dans la forêt classée de l'Ouémé Supérieur (127176 ha) à l'aide d'une machette.

Pour tester cette multiplication, deux classes de diamètre de bouture ont été cherchées [0 cm à $1,5 \mathrm{~cm}]$ et $[1,5 \mathrm{~cm}$ à $3 \mathrm{~cm}]$. Ces classes de diamètres peuvent s'enraciner 
correctement avec une grande capacité de retentions des subsistances pour les bougeons (Ouinsavi et al., 2019). Vingt-trois (23) boutures de tiges et 23 boutures de racines ont été utilisées pour chaque classe de diamètre soit au total 46 boutures de tiges et 46 boutures de racines. Le dispositif en randomisation total a été utilisé. Les traitements ont été ainsi distribués totalement au hasard par tirage aléatoire équiprobable. Les pots ont été remplis de sable forestier de la zone d'étude. Les tirages ont été faits à partir de 4 papiers portant le nom des 4 traitements (BtD1, BtD2, BrD1, $\mathrm{BrD} 2)$. Les boutures ont été piquées dans les pots immédiatement après les mesures de coupure avec sécateur. L'arrosage s'est fait avec une boîte d'une capacité de $33 \mathrm{cl}$ d'eau, deux fois par jour (1 le matin et 1 le soir) sauf en cas de pluie. Les boutures des deux classes ont été ombragées pour éviter la frappe des rayons solaires et les fortes pluies. Au cours de la conduite de l'essai, le nombre de bourgeons, le nombre bourgeons débourrées et les boutures desséchées ont été dénombrées chaque semaine. Les différents paramètres cidessus ont été calculés :

$\checkmark$ Les taux de bourgeonnement (Tb) par traitement, exprimés par le nombre de boutures ayant bourgeonné $(N b b)$ par rapport au nombre de boutures mises en terre $(N b t) . \boldsymbol{T} \boldsymbol{b}=\frac{N \boldsymbol{b} \boldsymbol{b}}{\boldsymbol{N} \boldsymbol{b} t} \boldsymbol{X} \mathbf{1 0 0}$

$\checkmark$ Les taux de débourrement (Td) par traitement, exprimés par le nombre de boutures ayant débourré (Nbd) par rapport au nombre de boutures mises en terre (Nbt). $T d=\frac{N b d}{N b t} X 100$

$\checkmark$ Les taux de survie (Ts) par traitement, exprimés par le nombre de boutures ayant gardé leurs feuilles (Nbf) à la fin de l'essai par rapport au nombre de boutures mises en terre (Nbt). $\quad T s=\frac{N b f}{N b t} X 100$

\section{Analyse statistique}

Pour tester l'effet de la grosseur des boutures et le type de bouture de $P$. kotschyi sur les paramètres étudiés (bourgeonnement, débourrement et taux de suivi), une analyse statistique de variance au seuil de $5 \%$ sous le logiciel R 3.2.4 a été faite. Les Histogrammes ont été réalisés pour montrer les taux de bourgeonnement et éclatement du bourgeon.

\section{RESULTATS}

\section{Taux de germination des différents traitements}

La Figure 1 présente le taux de germination et la dormance des graines de $P$. kotschyi. Elle a montré que les graines de $P$. kotschyi ont un bon taux de germination $(\mathrm{Tg}=$ 84\%). La germination des graines de $P$. kotschyi a démarrée $11^{\text {ème }}$ jours après la mise en terre. Cette germination a duré 19 jours (Figure 1).

\section{Multiplication végétative de $P$. kotschyi Bourgeonnement et débourrement des boutures de racine et de tige de P. kotschyi}

Le Tableau 1 présente le bourgeonnement des boutures de racine selon la grosseur des boutures. Son analyse montre que la grosseur des boutures de racine a un effet significatif $(\mathrm{P}<0,05)$ sur le suivi des bourgeons. Seules les boutures de racine de grand diamètre $[1,5 \mathrm{~cm}$ à $3 \mathrm{~cm}]$ ont données en moyenne 10,62 bougeons dont 6,12 de bourgeon ont débourrés par bouture. Le Tableau 2 présente le bourgeonnement des boutures de tige suivant la grosseur des boutures. De l'analyse du tableau, il ressort que la grosseur des boutures de tige a un effet statistique très significatif $(\mathrm{P}<0,05)$ sur le bourgeonnement des boutures et sur le débourrement des bourgeons. Les boutures de tige de diamètre [0 à $1,5 \mathrm{~cm}]$ et $[1,5 \mathrm{~cm}$ à $3 \mathrm{~cm}]$ ont donné respectivement une moyenne de 4,25 et 2,25 bougeons par bouture avec un taux de débourrement de 0,87 et 2,25 par bouture. Par ailleurs les boutures de petit diamètre ont perdu tous les bourgeons débourrés tandis que les boutures de grand diamètre ont gardé au moins un bourgeon débourré.

\section{Taux de réussite du bouturage par bouture de racine et de tige de $P$. kotschyi}

La Figure 2 présente le taux de réussite de bouturage $P$. kotschyi par bouture de racine. Il ressort de ce graphe que les boutures de racines de grosseur $\left[\begin{array}{lll}1,5 & \text { à } & 3 \mathrm{~cm}\end{array}\right]$ ont bourgeonnées à un taux de $73,91 \%$ avec un taux de débourrement de 52,17\%. Par ailleurs les boutures de racine de diamètre [0 à $1,5 \mathrm{~cm}]$ 
ont bourgeonnées à un taux de 56,52\% avec un taux de débourrement de $30,43 \%$. En termes de suivi, les boutures de racine de diamètre $[1,5$ à $3 \mathrm{~cm}]$ ont données un taux de suivi plus élevé $(47,82 \%)$ que les boutures de racine de diamètre [0 à $1,5 \mathrm{~cm}](21,74 \%)$. Les jeunes pousses issues des boutures de racine de grand diamètre telles qu'illustrée (Photo 1) présentent de très bonne caractéristique morphologique. La Figure 3 présente le taux de réussite de bouturage de $P$. kotschyi par bouture de tige. Les boutures de tiges de grosseur [0 à 1,5 cm] ont bourgeonnées à un taux de $17,39 \%$ pour un taux de débourrement de 4,35\%. Par contre, les boutures de tige de diamètre $[1,5 \mathrm{~cm}$ à $3 \mathrm{~cm}]$ ont bourgeonnées à un taux de $8,69 \%$ avec un taux de débourrement de $8,69 \%$. Les boutures de tige ont donné un taux de suivi de $0 \%$ quelle que soit la classe du diamètre de la bouture.

On observe un dessèchement total des boutures de tige (Photo 2. $\mathrm{b}_{2}$ ). Les jeunes poussent issus des boutures de tige sont sans tronc juste un allongement d'une feuille (Photo 2. $\left.b_{1}\right)$.

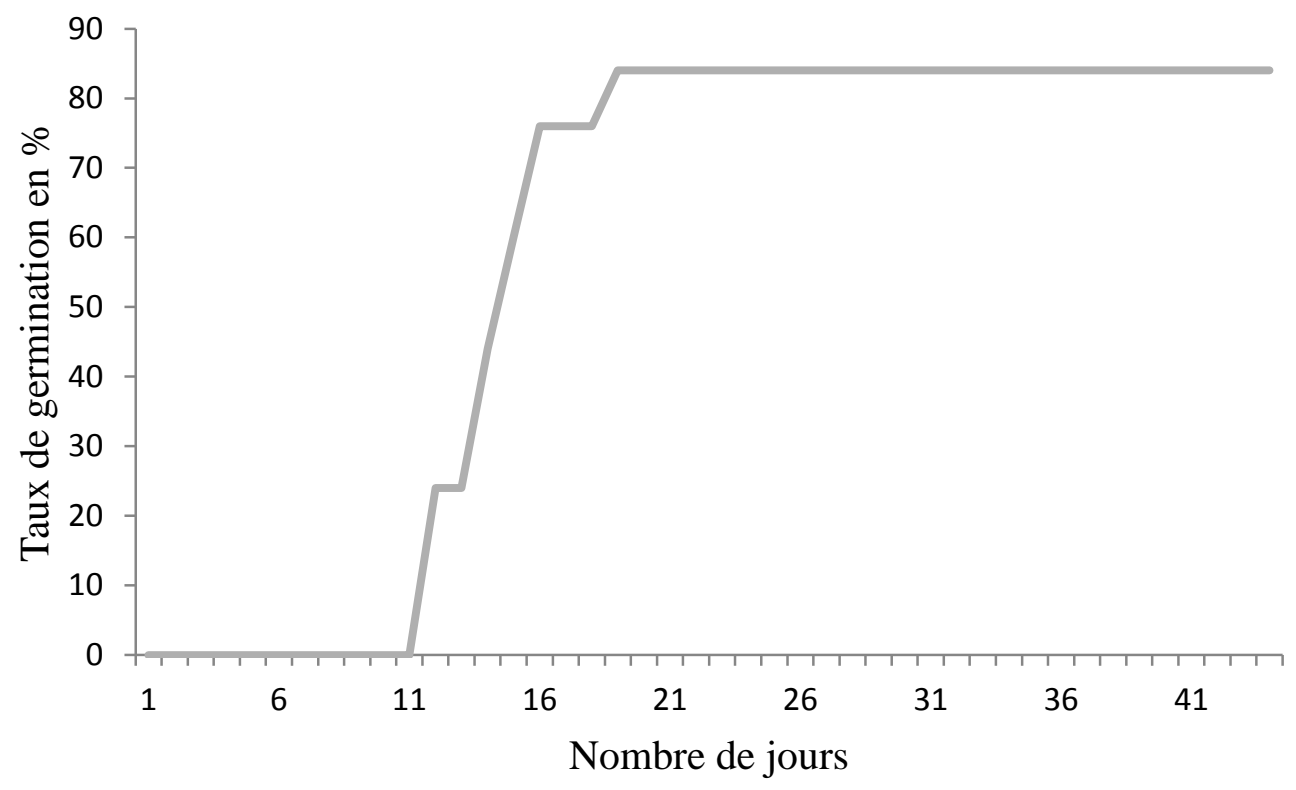

Figure 1: Germination des graines de $P$. kotschyi montrant le taux de germination, le temps de latence et la durée de germination.

Tableau 1: bourgeonnement et débourrement des boutures de racine.

\begin{tabular}{lcccc}
\hline Racine & Diamètre & F value & Pr(>F) & Moy. \\
\hline Nombre de bourgeon & D1 & 4,182 & 0,0601 & 6,50 \\
& D2 & & & 10,62 \\
\hline Nombre de bourgeon débourré & D1 & 4,115 & 0,062 & 2,87 \\
& D2 & & & 6,12 \\
\hline $\begin{array}{l}\text { Nombre de bourgeon mort/ taux } \\
\text { de suivi }\end{array}$ & D1 & 3,12 & $0,0442 *$ & 2,50 \\
& D2 & & & 5,62 \\
\hline
\end{tabular}

$\mathrm{F}$ value $=$ Ecart type $; \operatorname{Pr}(>\mathrm{F})=$ probabilité et *effet de significativité. 
Tableau 2: Bourgeonnement et débourrement des boutures de tige.

\begin{tabular}{lllll}
\hline Tige & Diamètre & F value & $\operatorname{Pr}(>\mathbf{F})$ & Moy. \\
\hline Nombre de bourgeon & D1 & 44,8 & $0,00^{* * *}$ & 4,25 \\
& D2 & & & 2,25 \\
\hline Nombre de bourgeon débourré & D1 & 44,58 & $0,00^{* * *}$ & 0,87 \\
& D2 & & & 2,25 \\
\hline Nombre de bourgeon mort & D1 & 2,333 & 0,149 & 0,25 \\
& D2 & & & 1 \\
\hline
\end{tabular}

F value $=$ Ecart type $; \operatorname{Pr}(>\mathrm{F})=$ probabilité $; * * *$ effet statistique très significatif.

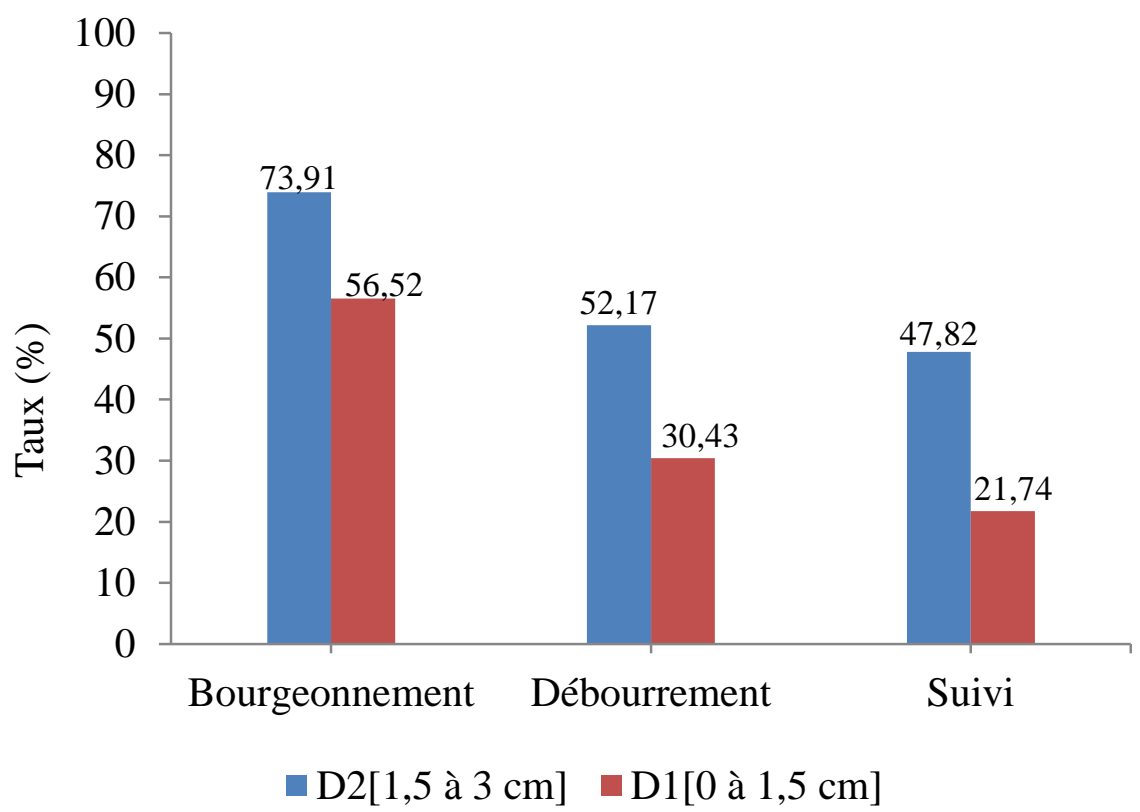

Figure 2: Le taux de réussite de bouturage $P$. kotschyi par bouture de racine.

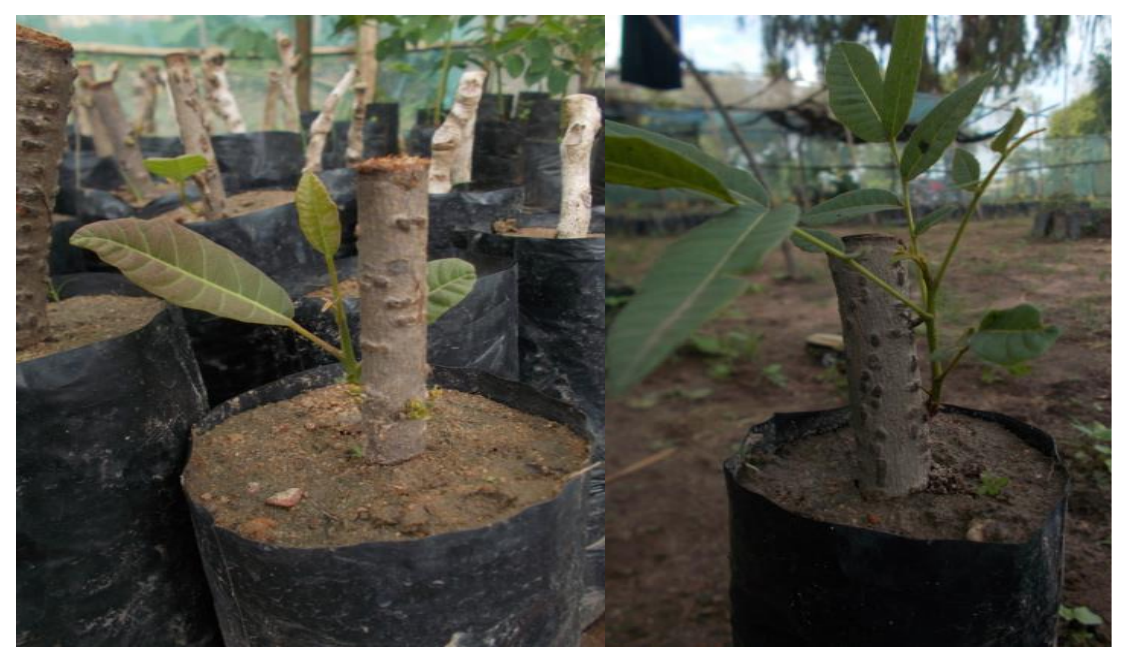

Photo 1: Etat de réussite du bouturage par bouture de racine. 


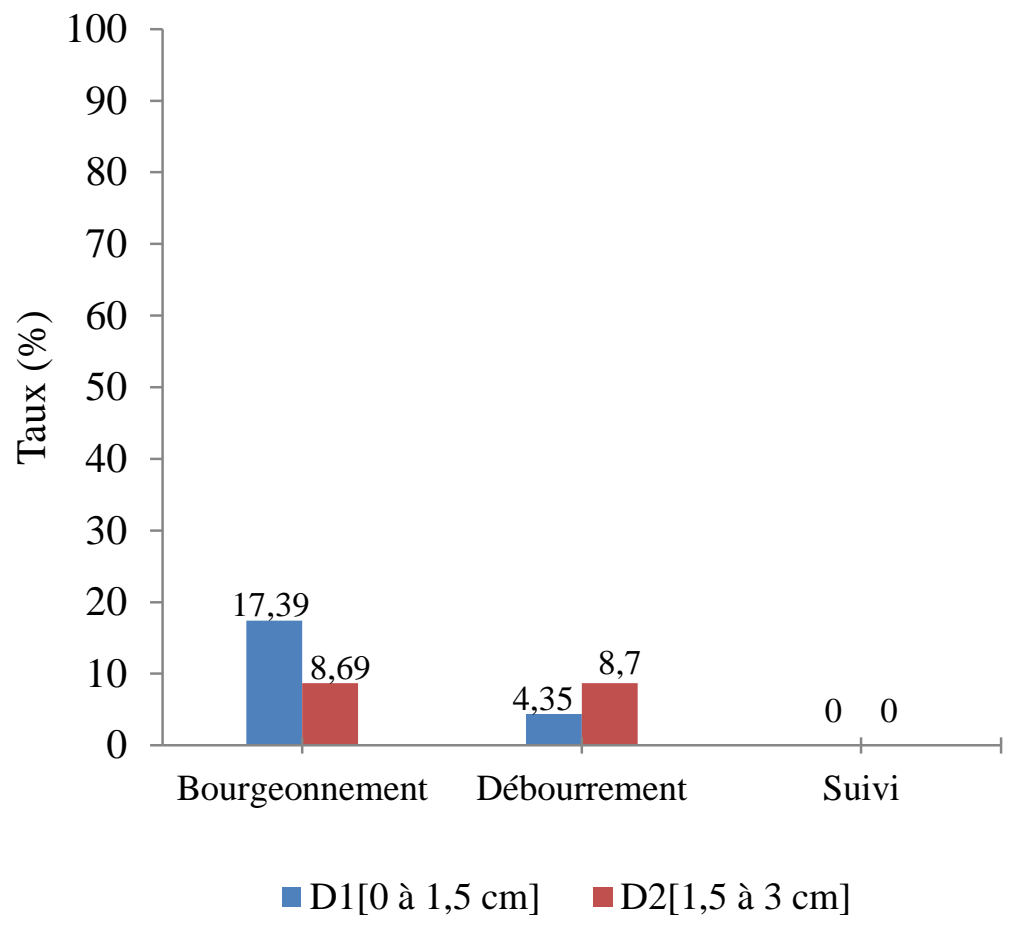

Figure 3: Le taux de réussite de bouturage $P$. kotschyi par bouture de tige.
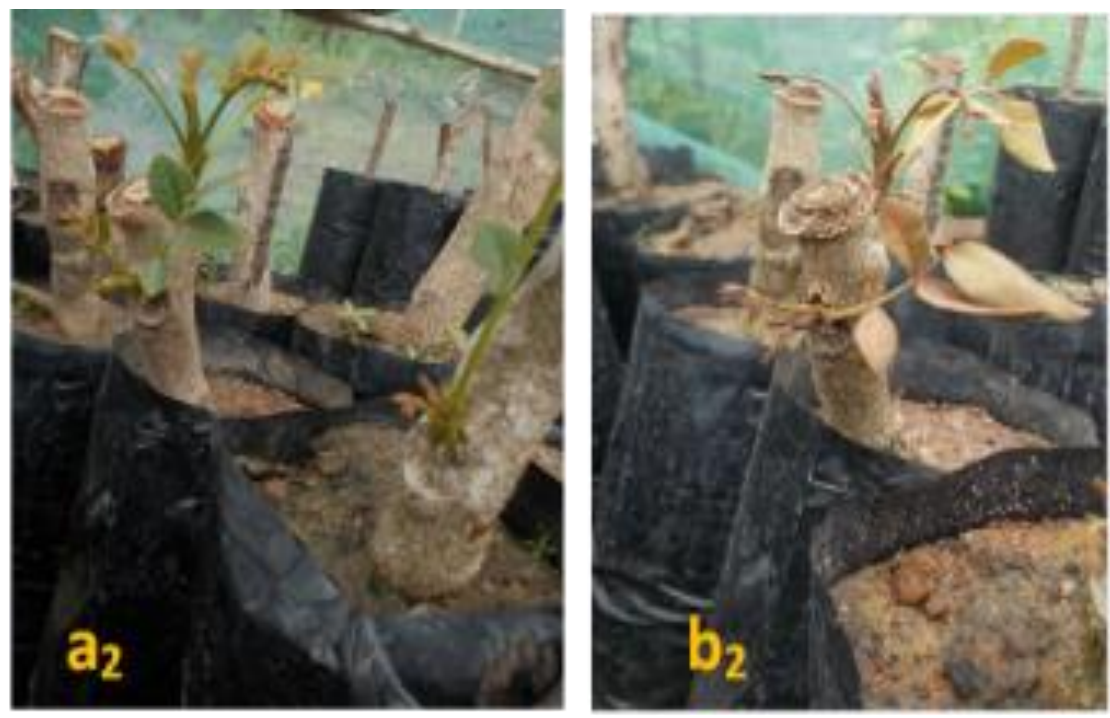

Photo 2: Illustration du débourrement $\left(a_{2}\right)$ et le dessèchement $\left(b_{2}\right)$ des boutures de tige. 


\section{DISCUSSION}

\section{Multiplication sexuée (graine) de $\boldsymbol{P}$. kotschyi}

Pour une meilleure conservation des semences, les graines de $P$. kotschyi ont été conservées pendant environ deux (02) mois dans un bocal perforé afin de favoriser la respiration et permettre à la graine de perdre un peu de sa teneur en eau. Car la teneur en eau est sans doute l'un des principaux paramètres qui affectent la longévité des semences. Ces résultats sont conformes à ceux obtenus par Babou et al. (2000) sur la longévité des graines de A. africana.

Plusieurs études ont été menées en ce qui concerne l'efficacité des différents traitements sur la germination des graines des essences forestières (Onyekwelu \& Akindele, 2002; Onyekwelu, 2004). Il s'agit des graines de Mansonia altissima qui présentent de fort taux de germination (81\%) après un traitement de trempage dans l'eau bouillante pendant 60 secondes (wédjangnon et al., 2016). Mais en ce qui concerne $P$. kotschyi, seules les graines sans traitement pré-germinatif ont donné un taux de germination élevé de l'ordre de $84 \%$ après dix-neuf (19) jours de mise en germination. Ces résultats pourraient bien s'expliquer par le fait que les graines de $P$. kotschyi ne disposent pas de téguments assez dur pouvant ralentir la germination. Malgré ce pouvoir germinatif élevé, $P$. kotschyi présente une probabilité de reconstitution très faible $(4,1 \%)$ dans les forêts claires du Bénin (Sokpon et al., 2006). Ce qui pourrait s'expliquer par la consommation des graines par les rongeurs, leur destruction par les feux de végétations et l'effet du piétinement lié au pâturage extensif. L'aménagement devra leur prêter une attention particulière.

\section{Multiplication végétative par bouture de racine et tige de $P$. kotschyi}

La multiplication végétative par bouture de racine montre un fort taux de réussite. Effet, seules les boutures de racine de grands diamètres $(1,5 \mathrm{~cm}$ à $3 \mathrm{~cm})$ ont donné plus de bourgeons débourrés avec un fort taux de suivi. Cela pourrait se justifier par l'existence d'une réserve nutritive importante de cette classe de diamètre. En exemple, le diamètre de coupe et la longueur des boutures affectent significativement le taux de régénération des boutures (Dako et al., 2014). Par contre, le bourgeonnement et le débourrement à eux seuls, n'expliquent pas les chances de réussite des jeunes pousses car celles-ci dépendent aussi bien des réserves nutritives que de l'enracinement des boutures (Ouinsavi et al., 2019).

La multiplication végétative par boutures de tiges présente un taux de bourgeonnement et de débourrement très faible avec un taux suivi presque nul. Ce faible taux de réussite de ces boutures de tige pourrait s'expliquer par une insuffisance de réserves nutritives dans les boutures. Ce constat a été fait par Traoré (2000) sur Piliostigma reticulatum que l'échec du bouturage des jeunes rameaux était dû au fait qu'ils ne contiennent pas assez de réserves nutritives nécessaires. Ce faible taux de réussite des boutures de tiges pourrait également s'expliquer par le fait qu'elles n'ont pas réussi l'étape de la cicatrisation. Ces résultats concordent avec ceux de Jaenicke et Beniest (2003) et Agbogan et al. (2014), qui ont montré que la cicatrisation est une étape clé du bouturage qui est influencée par plusieurs facteurs. Aussi, il a été constaté que les boutures de tige n'ont pas pu réussir l'étape d'enracinement ce qui pourrait justifier le faible taux de débourrement et de survie. En effet, après l'épuisement des réserves nutritives de la bouture de tige en absence d'un système racinaire qui peut assurer alimentation la jeune pousse, les boutures sèchent et meurent. $P$. kotschyi est alors une espèce qui ne répond pas à la voie de multiplicative par bouture de tige.

Malgré la réussite de la multiplication végétative par bouture de racine, le type de substrat utilisé semblerait aussi expliquer les 
faibles taux de réussite de cet essai car, il existe une relation entre la teneur en eau du substrat et celle de la bouture (Mapongmetsem et al., 2012).

\section{Conclusion}

Cette étude a permis d'évaluer les voix de multiplication de $P$. kotschyi par ses différents organes : graine, bouture de racine et de tige. Les résultats obtenus révèlent que $P$. kotschyi se multiplie par graine sans un traitement prégerminatif. La multiplication végétative par bouture de racine a montré de bonne performance de bourgeonnement, débourrement et de suivi des plantules. Au regard de ces résultats, la recherche des conditions de conservation des graines est une solution envisageable pour la conservation de l'espèce, dans la mesure où la production des plants en pépinière est possible pour enrichir les écosystèmes dégradés et, vu que le potentiel semencier de l'espèce dans les forêts classées est très faible. De même, la multiplication végétative par bouture de racine, bien ayant présenté de bonne performance de régénération (bourgeonnement, débourrement et suivi) mérite une attention particulière en ce qui concerne l'enracinement des boutures et la nature des substrats.

\section{CONFLIT D'INTERETS}

Les auteurs déclarent que l'article n'est sujet d'aucun problème ou d'intérêt concurrentiel.

\section{CONTRIBUTIONS DES AUTEURS}

Les auteurs de l'article ont participé chacun en ce qui le concerne à la mise au point du manuscrit. En effet, l'exécution du protocole et la rédaction ont été effectués DGA, l'analyse statistique des données ont été effectués par DJ. Enfin la lecture du manuscrit a été faite par IR.

\section{REFERENCES}

Adomou AC, Dassou GH, Houénon GHA, Alladayè A, Yedomonhan H. 2017. Comprendre les besoins en ressources végétales des populations riveraines pour une gestion durable de la forêt Bahazoun au Sud-Bénin (Afrique de l'Ouest). International Journal of Biology and Chemical Sciences, 11(5): 2040-2057. DOI: https://dx.doi.org/10.4314/ijbcs.v11i5.9

Agbani PO, Sinsin B, Hahn-Hadjal K. 2004. Phytodiversity Decline: The local perception in the soudan zone of norh West Bénin. 5th inter Symposium of Tropical African Biodiversity-Molecule, Ortganisme-ecosystem, 2-6.

Agbogan A, Bammite D, Tozo K, Akpagana K. 2014. Contribution à la multiplication, par graines et par bouturage de segments de tiges et de racines, de trois fruitiers spontanés de la région des savanes au Togo : Haematostaphis barteri Hook. F., Lannea microcarpa Engl, K. Krauss et Sclerocarya birrea (A. Rich.) Hochst. European Scientific Journal, 10 (6): 195211.

Akande JA, Hayashi Y. 1998. Potency of extract contents from selected tropical chewing sticks against Staphylococcus aureus and Staphylococcus auricularis. World J. Microbiol. Biotechnol., 14: 235238.

DOI: https://doi.org/10.1023/A:100883833107 9

Arbonnier M. 2009. Arbres, Arbustes et Lianes des Zones Sèches d'Afrique de l'Ouest. CIRAD-MNHN : Paris ; P.531.

Avikpo DJ, Dassou GH, Adomou AC, Houénon GHA, Tente B, Sinsin AB. 2017. Impact des caractéristiques de la végétation sur la diversité d'usages des plantes autour de deux grandes forêts classées et d'une réserve botanique au Sud-Bénin. European Scientific Journal, 
13(30): 376-394. DOI: 10.19044/esj.2017.v13n30p376

Babou AB, Sibiri JO, Sita G. 2000. Longévité des graines et contraintes à la survie des plantules d'Afzelia africana Sm. dans une savane boisée du Burkina Faso. Ann. For. Sci., 58: 69-75. https://hal.archivesouvertes.fr/hal-00883659

Bellefontaine R, Gaston A, Petrucci Y. 2000. Management of natural forest of dry tropical zones. Food and Agricultural Organization (FAO), Conservation Guide, ${ }^{\circ} 32$, Rome.

Goussanou C, Tenté B, Djègo J, Agbani P, Sinsin B. 2011. Inventaire, caractérisation et mode de gestion de quelques produits forestiers non ligneux du Bassin versant de la Donga. Annales Sciences Agronomiques, 14(1): 77-99.

Jaenicke H, Benies J. 2003. La multiplication végétative des ligneux en agroforesterie. Manuel de formation et bibliographie, World Agrofor. Cent. ICRAF ISBN 92 90591501 KulGraphics Ltd, Nairobi Kenya.

Kassim OO, Loyevsky M, Amonoo H, Lashley L, Ako-Nai KA, Gordeuk VR. 2009. Inhibition of in-vitro growth of Plasmodium falciparum by Pseudocedrela kotschyi extract alone and in combination with Fagara zanthoxyloides extract. Trans. R. Soc. Trop. Med. Hyg., 103: 698-702. DOI: 10.1016/j.trstmh.2009.02.018

Koné WM, Atindehou KK, Terreaux C, Hostettmann K, Traore D, Dosso M. 2004. Traditional medicine in North Côte d'Ivoire: Screening of 50 medicinal plants for antibacterial activity. Journal Ethnopharmacol., 93: 43-49. DOI: 10.1016/j.jep.2004.03.006

Mapongmetsem PM, Fawa G, Bellefontaine RR. 2012. Bouturage des segments racinaires de Vitex doniana Sweet. (Verbenaceae) : technique de multiplication végétative à faible coût. In:
International Symposium on Tree Product Value Chains in Africa. Source : Cirad (https://agritrop.cirad.fr/568159/)

Ministère de l'Environnement et de la Protection de la Nature. 2009. 4ème Rapport National du Bénin sur la Convention des Nations Unies sur la Diversité Biologique, PNUD/MEPN, Cotonou, Bénin, p 151.

Ojewole JAO. 2003. Evaluation of the antiinflammatory properties of Sclerocarya birrea (A. Rich.) Hochst. (family: Anacardiaceae) stem-bark extracts in rats. J. Ethnopharmacol., 85: 217-220. DOI: 10.1016/s0378-8741(03)00019-9

Okunade MB, Adejumobi JA, Ogundiya MO, Kolapo AL. 2007. Chemical, phytochemical compositions and antimicrobial activities of some local chewing sticks used in South Western Nigeria. J. Phytopharmacother. Nat. Prod., 1(1): 49-52.

Onyekwelu JC. 2004. Above-ground biomass production and biomass equations for even aged Gmelina arborea (Roxb) plantations in south-western Nigeria. Biomass Bioenergy, 26(1): 39-46. DOI: 10.1016/s0961-9534(03)00100-4

Onyekwelu JC, Stimm B. 2002. Gmelina arborea. In Enzyklopa"die der Holzgewächse $-28 \mathrm{Erg}$. Lfg. Ecomed Publishers : Munich, Germany.

Ouinsavi C, Sourou B, Houètchégnon T, Wédjangnon A, Dossa B, Akin Y, Dossou J, Houndjo M-R. 2019. Effect of cuttings diameter and indol acetic acid on rooting of Pterocarpus erinaceus Poir. stem cuttings. International Journal of Agroforestry and Silviculture, 7(10): 001010.

Projet Bois de Feu phase II/ Inventaire forestier National. 2007. Bassins d'approvisionnement en bois énergie de Cotonou, Porto Novo, Lokossa, Abomey, Bohicon, Djougou, Natitingou et 
Parakou, Rapport de mission de Claudine SERRE DUHEM Mission au Bénin. Ministère de l'Environnement et de la Protection de la Nature, Cotonou, Bénin. P.54.

Sokpon N, Biaou SH, Ouinsavi C, Hunhyet O. 2006. Bases techniques pour une gestion durable des forêts claires du Nord-Bénin : rotation, diamètre minimal d'exploitabilité et régénération. Bois et Forêts des Tropiques, 287(1): 45-57.

Traoré M. 2000. Etude de la phénologie, de la régénération naturelle, et des usages de Piliostigma reticulatum (de.) Hochst. en zone nord soudanienne du Burkina Faso. Mémoire d'Ingénieur du Développement Rural, Université Polytechnique de Bobo Dioulasso, p.87.

Wédjangnon AA, Houètchégnon T, Ouinsavi C. 2016. Ecological Characterization and
Mass propagation of Mansonia altissima A. Chev. in the Guinean Zone of Benin, West Africa. Int. J. Pure App. Biosci., 4(4): $\quad 15-25 . \quad$ DOI: http://dx.doi.org/10.18782/23207051.2339 .

Yaoitcha AS, Aboh AB, Zoffoun AG, Houinato M, Mensah GA, Sinsin B, AKPO EL. 2016. Potentiel de régénération des chantiers de production du charbon de bois au Centre-Bénin. Int. J. Biol. Chem. Sci., 10(4): 1702-1716. DOI http://dx.doi.org/10.4314/ijbcs.v10i4.21 Yaoitcha AS. 2007. Potentiel de régénération des chantiers de production du charbon de bois au Centre-Bénin. Mémoire de Diplôme d'Etude Approfondie, Faculté des Sciences Agronomiques, Université d'Abomey Calavi, Bénin. P.33. 